\title{
AVALIAÇÃO DE INTERFACE ACESSÍVEL PARA USUÁRIOS COM DEFICIÊNCIA VISUAL EM EMULADOR DE CONTROLE REMOTO PARA SMART TV
}

\section{AFFORDABLE INTERFACE ASSESSMENT FOR VISUALLY IMPAIRED USERS ON REMOTE CONTROL EMULATOR FOR SMART TV}

\author{
Luiz Henrique Bier Maia ${ }^{1}$, Bel. \\ Elton Moura Nickel ${ }^{2}$, Dr. \\ (1) Universidade do Estado de Santa Catarina - UDESC \\ e-mail: lhbmaia@gmail.com \\ (2) Universidade do Estado de Santa Catarina - UDESC \\ e-mail: eltonnickel@gmail.com
}

palavras chave: acessibilidade, smartphone, interface, controle remoto TV

Este artigo é uma introdução à pesquisa de aplicativos que emulam um controle remoto de Smart TV, a partir de dispositivo móvel de sistema operacional Android, pela perspectiva de usuários com deficiência visual. Metodologicamente é caracterizada por uma pesquisa hipotética-dedutiva, orientada pela apropriação teórica na esfera das recomendações de acessibilidade e usabilidade para dispositivos móveis, disponível na documentação do Google. A avaliação foi conferida por usuário cego, consorciada por uma checklist, adaptada da proposta do Governo Eletrônico Brasileiro (e-MAG). Verificou-se que este primeiro ensaio pode significar um estudo mais aprofundado na adequação e melhoria da acessibilidade em aplicativos para dispositivos móveis.

key words: Accessibility, smartphone, interface, TV remote control

This article is an introduction to researching applications that emulate a Smart TV remote control, from Android operating system mobile device, from the perspective of visually impaired users. Methodologically it is characterized by a hypothetical-deductive research, guided by theoretical appropriation in the sphere of the recommendations of accessibility and usability for mobile devices, available in the Google documentation. The avaliation was verified by blind user, consorted by a checklist, adapted from the proposal of the Brazilian Electronic Government (e-MAG). It has been found that this first trial may mean further study on the suitability and improvement of accessibility in mobile applications.

\section{Introdução}

O avanço da tecnologia na última década promoveu o

desenvolvimento e aprimoramento de ferramentas de acessibilidade para pessoas com deficiência. Isso trouxe 


\section{$16^{\circ}$ \\ ERGODESIGN USIHC CINAHPA}

$16^{\circ}$ Ergodesign - Congresso Internacional de Ergonomia e Usabilidade de Interfaces Humano Tecnológica: Produto, Informações Ambientes Construídos e Transporte

$16^{\circ}$ USIHC - Congresso Internacional de Ergonomia e Usabilidade de Interfaces Humano Computador

CINAHPA | 2017 - Congresso Internacional de Ambientes Hipermídia para Aprendizagem. uma melhora na qualidade e ampliação do acesso à informação e nas capacidades comunicativas, com a consequente inclusão da pessoa com deficiência (PCD) na sociedade, exercendo sua cidadania. A utilização de softwares de acessibilidade em desktops e smartphones permitiu a essas pessoas executar de forma mais independente as tarefas comuns cotidianas. Os dispositivos móveis têm ganhado protagonismo global, tanto na comercialização de aparelhos e

desenvolvimento de aplicativos, quanto na maior aceitação por parte do público cego. Segundo pesquisa realizada no Brasil, em 2010 foram comercializados 125 milhões de unidades, aproximadamente quatro vezes mais que os computadores. No $3^{\circ}$ trimestre de 2013 , ocorreu uma alta de $147 \%$ no número de vendas de smartphones (10,4 milhões de unidades vendidas). Estima-se que em 2016 serão 168 milhões de unidades comercializadas. Houve uma queda nas vendas, porém com um aumento de $9 \%$ em relação a 2015. As projeções são de que, embora um pouco contidas se relacionadas com 2013, as vendas de dispositivos móveis em 2018 cheguem ao patamar de 236 milhões de unidades vendidas. A popularidade dos dispositivos móveis, além do fator financeiro, é compreendida também pela maior interatividade que promove. As telas touchscreen ampliam e simplificam as interações com a interface, reduzindo o tempo de resposta. Com essa potencialização de interação, em conjunto com as ferramentas de acessibilidade nativas dos dispositivos móveis, o número de usuários com deficiência tem aumentado exponencialmente. Evidentemente que no caso de operações mais complexas, como por exemplo produção textual, o uso de desktop ou notebook torna-se a escolha mais adequada.

As smart TVs compreendem um grupo de televisões que, através da conexão Wi-Fi ou via cabo, une a função básica dos aparelhos com o acesso à web, acesso a conteúdo pessoal, instalação de aplicativos e diversos outros itens que melhoram a experiência de uso. Na Smart TV, a operacionalização é realizada através de controle remoto, visto que o único botão presente no corpo do aparelho é o liga/desliga. Sendo o controle remoto o instrumento de interação entre usuário e TV, é importante observar que os controle remotos são projetados para pessoas videntes, com uso de cores e tamanhos de botão diferentes para facilitar o manuseio. Devido ao grande número de funcionalidades, sua interface torna-se complicada até para quem é vidente. Segundo o Design Universal, “os produtos devem ser projetados para que possam ser utilizados por todas as pessoas, de maneira fácil, reduzindo as possibilidades de erro." No caso de um usuário cego ou baixa visão, a utilização da TV por meio do controle remoto torna a atividade altamente frustrante, pela falta de elementos acessíveis para a sua utilização. No entanto, as maiores fabricantes de Smart $T V$ mundiais já oferecem aplicativo para dispositivos móveis que emulam o controle remoto, podendo assim a pessoa cega operar a TV através uso de Smartphone.

Dessa forma, confrontando os problemas de acessibilidade observados no uso de controle remoto físico, e na possibilidade de discutir a respeito da sua substituição por um emulador instalado em Smartphone, o foco da investigação proposto nessa pesquisa foi orientado pela seguinte questão: o uso de um emulador de controle remoto pode tornar mais acessível a experiência de um usuário com limitações visuais?

\section{USABILIDADE E ACESSIBILIDADE}

A norma ISO 9241-11 [2002, p. 3] define que USABILIDADE é "a capacidade que um sistema interativo oferece a seu usuário, em determinado contexto de operação, para a realização de tarefas com eficácia, eficiência e satisfação em um contexto de uso específico." Dessa forma, para melhor compreensão, esclarece seus conceitos: Usuário - pessoa que interage com o produto; Contexto de uso - usuários, tarefas, equipamentos (hardware, software e materiais), ambiente físico e social em que o produto é usado; Eficácia - precisão e completeza com que os usuários atingem objetivos específicos, acessando a informação correta ou gerando os resultados esperados; Eficiência precisão e completeza com que os usuários atingem seus objetivos, em relação à quantidade de recursos gastos; Satisfação - conforto e aceitabilidade do produto. O produto deve proteger o seu usuário de possíveis erros de entrada e situações indesejáveis. Os princípios de usabilidade estão diretamente ligados à acessibilidade, e devem ser observados como itens importantes na concepção de novos produtos. No campo digital, a acessibilidade promove a adaptação de dispositivos eletrônicos e softwares desenvolvidos para atender aos mais diversos tipos de limitações físicas e intelectuais. [SANTANA, 2015]

Realização:




\section{$16^{\circ}$ \\ ERGODESIGN USIHC CINAHPA}

$16^{\circ}$ Ergodesign - Congresso Internacional de Ergonomia e Usabilidade de Interfaces Humano Tecnológica: Produto, Informações Ambientes Construídos e Transporte

$16^{\circ}$ USIHC - Congresso Internacional de Ergonomia e Usabilidade de Interfaces Humano Computador

CINAHPA | 2017 - Congresso Internacional de Ambientes Hipermídia para Aprendizagem.
Existem diversas abordagens e aplicações para o termo acessibilidade. Para a NBR 15290 [2005, p. 2], "ACESSIBILIDADE é a possibilidade e condição de alcance para utilização de meio físico, meios de comunicação, produtos e serviços por pessoas com deficiência. Qualquer entrave ou obstáculo que dificulte ou impossibilite a expressão ou o recebimento de mensagens por intermédio dos meios ou sistemas de comunicação, sendo ou não de massa." No contexto dessa pesquisa, para Rogers et al. [2013, p. 16] a acessibilidade digital "se refere ao grau em que um produto interativo é acessível para tantas pessoas quanto possível." Para Santana [2015, p. 23], a Tecnologia Assistiva (TA) "refere-se ao conjunto de recursos e serviços que colaboram no oferecimento e ampliação de habilidades com o intuito de promover a inclusão e tornar independente o modo de viver de uma PCD. Portanto o objetivo da TA é ampliar a comunicação, mobilidade, controle de ambiente, habilidades de aprendizado e trabalho de uma PCD, beneficiando as mesmas com qualidade de vida, inclusão social e maior independência." Explica ainda que "recursos são itens, equipamentos, produtos ou sistemas fabricados sob medida de modo a melhorar ou manter as habilidades funcionais de PCD. Serviços são os que de forma direta ajudam as PCD a escolher, adquirir ou utilizar os recursos. Por exemplo, avaliações, experimentação e treinamento de novos equipamentos."

O World Wide Web Consortium W3C é um órgão internacional que tem a iniciativa de auxiliar os desenvolvedores de sites para a web, fornecendo informações e diretrizes que alcem seus produtos ao potencial máximo de acessibilidade. Os quatro princípios apontados nas recomendações de acessibilidade apresentadas no documento $W C A G$ (Web Content Acessibility Guidelines) são: perceptível, operável, compreensível, e robusto. Apesar de serem concebidas para a web, esses princípios podem ser facilmente aplicáveis aos produtos desenvolvidos para plataformas móveis.

Para fins práticos, por tratar-se de um aplicativo para dispositivos móveis, serão observadas as recomendações de acessibilidade do Google, indicadas para projetos especificamente desenvolvidos para o sistema operacional Android. Em sua documentação oficial, a acessibilidade aparece como subitem de interface de usuário, com o título Making Applications Accessible, onde orienta os desenvolvedores de aplicativos a observarem quatro recomendações para o projeto de interfaces: (1) descrever os elementos da interface, com atenção especial para ImageButton, ImageView e CheckBox, utilizando o atributo android: contentDescription; (2) verificar se todos os componentes de interface de entrada de dados, toque ou digitação, podem ser acessados por meio de controle direcional - TrackBall ou D-pad (físico ou virtual) -, ou navegação por gestos; (3) Certificar-se de que prompts de áudio estejam acompanhados por elementos de descrição visual ou de notificação (usuários surdos); (4) testar a acessibilidade do aplicativo, ativando o TalkBack (leitor de tela) e utilizando as funcionalidades de navegação do dispositivo, por toque e por controles direcionais.

Segundo Silveira [2014, p. 70], "a informação tem que ser perceptível, chegando ao usuário de forma eficaz, independentemente do ambiente ou das suas capacidades sensoriais. Em relação aos componentes da interface com os usuários e com a navegação no sistema, estes devem ser fáceis de utilizar e compreender, de acordo com a capacidade e conhecimento de cada pessoa."

Por discutir um problema pela ótica da observação e da experimentação, o que condiciona esse estudo a uma pesquisa hipotética-dedutiva, o objeto de análise foi definido como um emulador de controle remoto para TV, instalado e sincronizado a partir de um dispositivo móvel com sistema operacional Android. Os fatores que orientaram a escolha do sistema operacional foram: (1) oferta no mercado; (2) ferramentas de acessibilidade nativos. $\mathrm{O}$ emulador escolhido é específico para os modelos de uma marca multinacional de aparelhos eletroeletrônicos e sua escolha foi orientada por: (1) oferta no mercado (gratuidade); (2) compatibilidade com o aparelho de TV.

\section{Acessibilidade no emulador de controle remoto para $\mathrm{TV}$}

A pesquisa foi estruturada na verificação e a validação dos requisitos de acessibilidade, pela utilização do recurso de acessibilidade TalkBack, nativo do sistema operacional Android, que traduz 


\section{$16^{\circ}$ \\ ERGODESIGN USIHC CINAHPA}

$16^{\circ}$ Ergodesign - Congresso Internacional de Ergonomia e Usabilidade de Interfaces Humano Tecnológica: Produto, Informações Ambientes Construídos e Transporte

$16^{\circ}$ USIHC - Congresso Internacional de Ergonomia e Usabilidade de Interfaces Humano Computador

CINAHPA | 2017 - Congresso Internacional de Ambientes Hipermídia para Aprendizagem. em informações sonoras os componentes da interface gráfica. Foi denominada Análise de Acessibilidade, que compreende as tarefas simples, como mudar canais e volume e as tarefas mais complexas, como acessar configurações e conteúdo interativo.

\section{Processo de Análise de Acessibilidade}

Nesse processo metodológico, utilizou-se a avaliação com usuário cego, por meio de uma checklist. Para Sonza [2013], checklist consiste em um documento com itens de verificação e acompanhamento detalhado, com o objetivo auxiliar na documentação dos testes manuais feitos por pessoas com deficiência e programadores. A referência utilizada para a construção da checklist foi o documento do Governo Federal Brasileiro para teste de acessibilidade na web [e-MAG, 2016], devidamente adaptado para dispositivos móveis. Para a adaptação, acrescentou-se os itens para avaliação: tempo de resposta do leitor de tela, resposta da navegação por imagens, referência de som ao reconhecer elementos da interface e a leitura dos elementos em forma de lista. $\mathrm{O}$ checklist também registra possíveis sugestões de melhorias ao sistema. O sujeito avaliador é diagnosticado com cegueira total (Sem Percepção de Luz - SPL), tem experiência e habilidade com dispositivos móveis e telas sensíveis ao toque. Utiliza o smartphone para tarefas cotidianas simples, como acessar redes sociais, gerenciar email, buscas rápidas na internet e faz uso de alguns aplicativos,como @Voice (leitor de PDF), 99 Taxis (transporte urbano). No teste solicitou-se ao avaliador desempenhar as seguintes ações: ligar o aparelho, buscar canais, gerenciar volume, acessar configurações, acessar conteúdo interativo.

Findado o processo de verificação e a avaliação efetuados no processo de Análise de Acessibilidade, a primeira observação é da efetiva importância da etiquetagem dos elementos de interface, em caso mais específico e fator mais importante, que resultaria na eficácia do serviço prestado pelo emulador, os botões. Os botões de operações básicas, como VOLUME (+/-), CANAIS (+/-) ou no botão OK, que confirma uma ação selecionada, não apresentam qualquer etiquetagem, sendo interpretados pelo TalkBack como "botão sem marcador". O sujeito avaliador gastou um tempo considerável para conseguir interpretar o rodapé fixo, em tentativa e erro. O rodapé é formado por uma linha de sete círculos que indicam a posição do usuário em relação às telas do emulador; na última linha, ao centro, o ícone MUDO, com os índices + (à direita) e - (à esquerda) como controladores de volume; no canto esquerdo o ícone CASA (que volta para a primeira tela), e no canto direito o ícone FORÇA (liga/desliga o dispositivo primário TV). Não há etiquetagem em nenhum dos botões. Do ponto de vista da funcionalidade, executam suas devidas ações, porém a confirmação se dá somente na tela do dispositivo primário (TV), que não possui qualquer tipo de leitor de tela para auxiliar na confirmação das ações.

Avaliação Manual De Acessibilidade - CHECKLIST

Aplicativo: emulador de controle remoto para Smart TV

Sistema Operacional: Android 4.1

Leitor de tela: TalkBack

\begin{tabular}{|c|c|c|}
\hline Pergunta Orientadora & $\begin{array}{c}\text { Fatos revelados } \\
\text { na verificação }\end{array}$ & Observações \\
\hline \multicolumn{3}{|c|}{ Botões } \\
\hline $\begin{array}{l}\text { Remetem para local } \\
\text { ao qual se propõem? }\end{array}$ & $\begin{array}{c}\text { Não. Todos os comandos } \\
\text { são efetivados no aparelho } \\
\text { de TV }\end{array}$ & $\begin{array}{l}\text { Sugestões } \\
\text { propostas }\end{array}$ \\
\hline Há descrição? & $\begin{array}{l}\text { Somente nos botões } \\
\text { de configuração }\end{array}$ & $\begin{array}{c}\text { Incluir } \\
\text { etiquetagem }\end{array}$ \\
\hline $\begin{array}{l}\text { A descrição é } \\
\text { adequada? }\end{array}$ & Aos que contém, sim & $\begin{array}{l}\text { Sugestões } \\
\text { propostas }\end{array}$ \\
\hline \multicolumn{3}{|c|}{ Imagens } \\
\hline Há descrição? & Não & \\
\hline $\begin{array}{c}\text { A descrição é } \\
\text { adequada? }\end{array}$ & Não & \\
\hline $\begin{array}{l}\mathrm{O} \text { toque nas imagens } \\
\text { executa alguma ação? }\end{array}$ & Não & \\
\hline \multicolumn{3}{|c|}{ Textos } \\
\hline $\begin{array}{c}\text { Apresentam a leitura } \\
\text { correta? }\end{array}$ & Sim & Funcional \\
\hline \multicolumn{3}{|c|}{ Elementos de Interface } \\
\hline $\begin{array}{c}\text { Os elementos de } \\
\text { interface foram } \\
\text { programados de forma } \\
\text { adequada? }\end{array}$ & $\begin{array}{c}\text { Não interferem de forma } \\
\text { negativa na leitura } \\
\text { pelo TalkBack }\end{array}$ & Funcional \\
\hline \multicolumn{3}{|c|}{ Leitor de Tela } \\
\hline $\begin{array}{l}\text { Revela um tempo } \\
\text { adequado para } \\
\text { os componentes } \\
\text { de elementos? }\end{array}$ & Sim & $\begin{array}{l}\text { Funcional e } \\
\text { acessível }\end{array}$ \\
\hline \multicolumn{3}{|c|}{ Listas } \\
\hline $\begin{array}{l}\text { A apresentação dos } \\
\text { elementos em listas } \\
\text { revela-se funcional } \\
\text { e acessível? }\end{array}$ & Não há presença de listas & \\
\hline
\end{tabular}

Tabela 1 - Checklist para avaliação de acessibilidade 
$16^{\circ}$ USIHC - Congresso Internacional de Ergonomia e Usabilidade de CINAHPA Interfaces Humano Computador

CINAHPA | 2017 - Congresso Internacional de Ambientes Hipermídia para Aprendizagem.

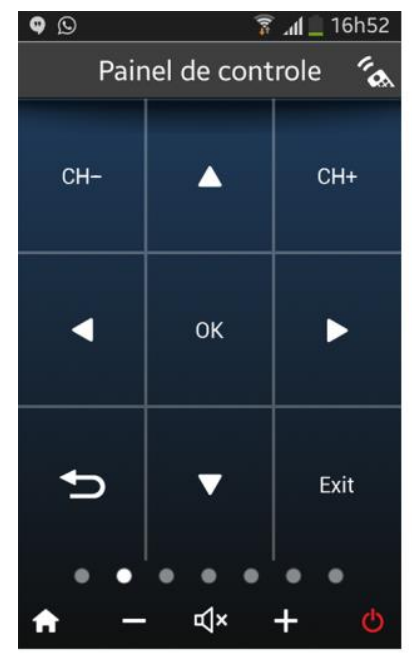

tela 1

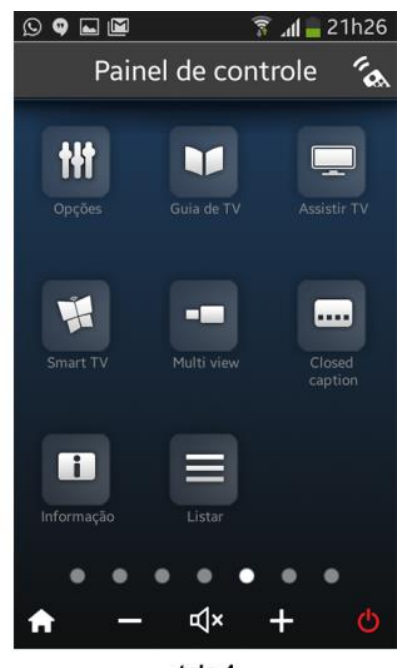

Figura 1 - Tela 1 e Tela 4

A tela 1 (figura 1) mostra os comandos básicos, em grade de 3x3: $\mathrm{CH}+$ (canal acima), SETA

DIRECIONAL ACIMA, CH- (canal abaixo), SETA DIRECIONAL ESQUERDA, OK (confirma ação selecionada), SETA DIRECIONAL DIREITA, RETORNO, SETA DIRECIONAL ABAIXO, EXIT (sair). Não há etiquetagem, o que dificultou a operacionalização das funções básicas pelo sujeito avaliador. A tela 2 apresenta no topo quatro botões sinalizados por cores distintas, que efetuam ações definidas como INFO ou AJUDA. Ao centro, em grade de $3 \times 4$ os números 1, 2, 3, 4, 5, 6, 7, 8, 9, ícone LISTA, zero, ponto. Somente os números são etiquetados mas não possuem descrição de função, sendo considerados mais como legendas. A tela 3 mostra os comandos para execução de programação "on demand", em grade de 3x3 com os ícones GRAVA, PARA, RETORNA CAPÍTULO, PAUSA, AVANÇA CAPÍTULO, RETORNO RÁPIDO, TOCA, AVANÇO RÁPIDO. Novamente, não há etiquetagem. As telas 4 (figura 1), 5 e 6 apresentam as funções de configurações avançadas do dispositivo primário e acesso ao conteúdo interativo. Todos os botões aqui estão etiquetados, porém não direcionam a lugar algum no emulador. A efetividade das ações executadas só são visíveis no dispositivo primário. $\mathrm{O}$ sujeito avaliador não encontrou dificuldades para achar as funções de configuração, mas ao acessá-las, perdeu a autonomia para completar a ação, pois o feedback é dado não através do emulador, e sim pela tela do dispositivo primário, que aguarda a confirmação da ação. Da mesma forma acontece ao acessar o conteúdo interativo: ao emulador cabe tão somente navegar pelas opções apresentadas na tela do dispositivo primário. $\mathrm{O}$ sujeito avaliador declara que "a gente acha que vai conseguir chegar em algum lugar, mas fica no meio do caminho, sem saber mais onde está. É igual ao controle físico, nesse caso"

Não há problemas relatados quanto aos elementos de estilização da interface, como os fios separadores, por exemplo. No rodapé, onde há os indicadores de posicionamento quanto às telas do emulador, o sujeito avaliador conseguiu compreender a mecânica de navegação por tentativa e erro. Sugere que os indicadores possam ser etiquetados de forma simples, como TELA 1, TELA 2, etc. e dessa forma diminuir o tempo gasto na varredura das telas, quando constrói um mapa mental do aplicativo. Não há utilização de navegação por listas nesse aplicativo, o que seria previsto quando se navega por opções de configuração, por exemplo. Portanto não se verificou funcionalidade, tampouco acessibilidade. Da mesma forma o emulador não faz uso de imagens, sendo nula sua avaliação nesse quesito.

\section{Considerações finais}

Ao longo dessa pesquisa foi possível observar a importância da realização de testes de avaliação de acessibilidade e, principalmente a sua validação através de usuários com limitações visuais. As recomendações de acessibilidade para a construção de sites e aplicativos propostas pelo Google promove a integração, comprometimento e interação entre desenvolvedores e usuários finais, e estão em consonância com as recomendações do documento W3C para a inclusão digital.

Com relação ao aplicativo avaliado, foi observado que: (1) a primeira recomendação (descrição dos elementos de interface) não foi completamente alcançada. A simples etiquetagem correta de botões pode significar um serviço mais acessível, dada a limitação estrutural do aplicativo. (2) a utilização do smartphone como ferramenta mediadora de acessibilidade e inclusão digital ganha credibilidade e aceitação por parte das pessoas com limitações visuais à passos largos, dado que amplia-se a capacidade de interação ao eliminar o uso de periféricos como teclado ou mouse. $\mathrm{O}$ recurso TalkBack nativo do
Realização:
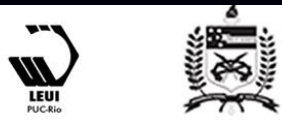


\section{$16^{\circ}$ \\ ERGODESIGN USIHC CINAHPA}

$16^{\circ}$ Ergodesign - Congresso Internacional de Ergonomia e Usabilidade de Interfaces Humano Tecnológica: Produto, Informações Ambientes Construídos e Transporte

$16^{\circ}$ USIHC - Congresso Internacional de Ergonomia e Usabilidade de Interfaces Humano Computador

CINAHPA | 2017 - Congresso Internacional de Ambientes Hipermídia para Aprendizagem.
Sistema Operacional Android revelou-se ferramenta robusta e bastante eficaz na operacionalização da acessibilidade. (3) a limitação estrutural do emulador faz como que este seja apenas uma ferramenta mediadora de botões, tornando-a uma simples virtualização do controle remoto físico e pouco interativa.

Este primeiro ensaio revela que a discussão acerca do tema ainda tem bastante campo a percorrer, abre caminho para que sejam realizados testes mais amplos, com outros emuladores e sistemas operacionais, afim de que possamos promover mais parcerias $\mathrm{e}$ comprometimento com desenvolvedores, e assim construírmos produtos mais acessíveis, conforme sugere o Design Universal. A inclusão de usuário cego no processo de avaliação traz a real possibilidade de consertar eventuais equívocos e prever outros, afim de promover a construção de produtos mais inclusivos, conforme testes de usabilidade e acessibilidade.

\section{BIBLIOGRAFIA}

Governo Eletrônico. Programa eMAG - Modelo de Acessibilidade de Governo Eletrônico. Disponível em: <http://emag.governoeletronico.gov.br/>. Acesso em: novembro de 2016.

GUIMARÃES, Ana Paula Nunes; TAVARES, Tatiana Aires. Avaliação de Interfaces de Usuário voltada à Acessibilidade em Dispositivos Móveis: boas práticas para experiências de usuário. In: IX Workshop de Testes e Dissertações (WTD) do XX Simpósio Brasileiro de Sistemas Multimídia e Web: João Pessoa, 2014.

ISO-9241. Ergonomic requirements for office work with visual displayterminals, Part 11 Usability Statements; Draft International Standard, 1998.

MACHADO, Douglas Ritter; MACHADO, Rodrigo Prestes; CONFORTO, Débora. Dispositivos móveis e usuários cegos: recomendações de acessibilidade em discussão. Nuevas Ideas en Informática Educativa TISE, 2014.

Making Application Accessible. Disponível em: $<$ http://developer.android.com/guide/topics/ui/accessibil ity/apps.html>. Último acesso em: novembro de 2016.
SANTANA, Ruan Domaria; BORGES, Thyago

Bohrer. Acessibilidade utilizando tecnologias assistivas para o uso de celulares ou computadores.In: Resumos Expandidos Apresentados no XIX Salão de Iniciação Cientifica, 2015.

SILVA, Giorgio Gilwan da et al. Diretrizes de acessibilidade para deficientes visuais a programação da TV digital interativa: contribuições, 2011.

SILVEIRA, Icléia; DA SILVA, Giorgio Gilwan. Princípios da usabilidade e da acessibilidade a serem aplicados na TV para facilitar o acesso aos deficientes visuais. e-Revista LOGO, v. 3, n. 2, p. 53-72, 2014.

SONZA, Andrea Poletto. et al. Acessibilidade e tecnologia assistiva: pensando a inclusão sociodigital de PNEs. Série Novos Autores da Educação Profissional e Tecnológica. Bento Gonçalves: Instituto Federal do Rio Grande do Sul Campus Bento Gonçalves/SETEC-MEC, 2013.

ROGERS, Y.; SHARP, H.; PREECE, J. Design de Interação: Além da Interação Humano-

Computador. Tradução de Isabela Gasparini. 3rd Edition. ed. Porto Alegre: Bookman Editora, 2013.

TS FILHO, Antonio Gerard; SANTOS, Gabriele MS. A ampliação da percepção da interface para pessoas com deficiência visual através de recursos em smartphones: Uma perspectiva do design da informação sobre a acessibilidade digital. Blucher Design Proceedings, v. 2, n. 2, p. 1239-1250, 2015.

WEB ACCESSIBILITY INITIATIVE, C2010. Disponível em: <http://www.w3.org/WAI/>. 2008. Acesso em novembro de 2016.

WCAG: Web Content Accessibility Guidelines2.0, C2011. Disponível em:

<http://www.w3.org/TR/WCAG20/>. Acesso em novembro de 2016.

\section{Agradecimentos}

Ao voluntário que dispôs-se a executar o teste para a realização dessa pesquisa, ao Programa de PósGraduação em Design CEART/UDESC e demais educadores envolvidos direta ou indiretamente, obrigado!
Realização:

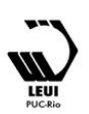

